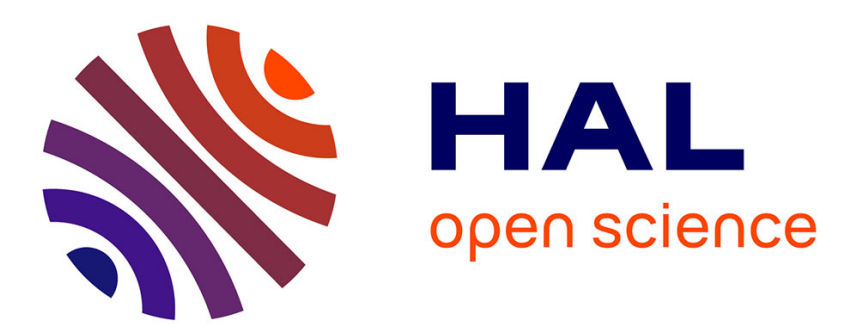

\title{
What eye movements reveal about strategy encoding of words in younger and older adults
}

Laurence Taconnat, Shasha Morel, Lina Guerrero Sastoque, Mickaël Frasca, N. Vibert

\section{- To cite this version:}

Laurence Taconnat, Shasha Morel, Lina Guerrero Sastoque, Mickaël Frasca, N. Vibert. What eye movements reveal about strategy encoding of words in younger and older adults. Memory, 2020, 28 (4), pp.537-552. 10.1080/09658211.2020.1745848 . hal-03090208

\section{HAL Id: hal-03090208 \\ https://hal.science/hal-03090208}

Submitted on 29 Dec 2020

HAL is a multi-disciplinary open access archive for the deposit and dissemination of scientific research documents, whether they are published or not. The documents may come from teaching and research institutions in France or abroad, or from public or private research centers.
L'archive ouverte pluridisciplinaire HAL, est destinée au dépôt et à la diffusion de documents scientifiques de niveau recherche, publiés ou non, émanant des établissements d'enseignement et de recherche français ou étrangers, des laboratoires publics ou privés. 


\title{
What eye movements reveal about strategy encoding of words in
}

\section{younger and older adults}

\author{
Laurence TACONNAT (corresponding author) \\ Phone: + 33 (0)247368154, laurence.taconnat@univ-tours.fr \\ Shasha MOREL \\ Phone: + 33 (0)683834571, morelshasha@yahoo.fr \\ Lina GUERRERO - SASTOQUE \\ Phone: + 33 (0)695542029, lina.guerrero@univ-tours.fr \\ Mickaël FRASCA* \\ Phone: + 33 (0)686361803, mikael.frasca@ hotmail.fr \\ Nicolas VIBERT \\ Phone: + 33 (0)549454634, nicolas.vibert@univ-poitiers.fr
}

Centre de Recherches sur la Cognition et l'Apprentissage, Université de Poitiers, Université de Tours, CNRS UMR 7295 ; Maison des Sciences de l’Homme et de la Société, Poitiers, France.

*Current workplace address: Nursing home « Le Belvédère Sainte-Clothilde », 2 rue du Moulin Neuf, 86190 Vouillé, France

Address for correspondence : Laurence Taconnat, Centre de Recherches sur la Cognition et 1'Apprentissage, 3 rue des Tanneurs, 37041 Tours cedex, France.

Running head: Eye-tracking and organizational strategies 
Eye-tracking and organizational strategies

\begin{abstract}
The main goal of this study was to explore the organizational strategies used by younger and older adults when encoding words, using eye-tracking. Participants had to learn a set of organizable words and then a set of non-organizable words, each presented on a single display. Participants were then asked to recall the words of each set in the order in which they came to their mind. Hence, the participants' encoding strategies revealed by eye-tracking could be directly related to their subsequent memory performance. The results confirmed the detrimental impact of aging on memory and the weaker use of organizational strategies by older adults during the recall phase. The eye-tracking data showed that when they encode the words, older adults do not look at them for as long as younger adults, probably because of slower eye movements. They also revealed that compared to younger adults, older adults were much less able to adapt their word scanning strategy according to whether the words to encode were organizable or not. Finally, the relationships that were found between the recall scores and the eye-tracking data suggest that the eye movement pattern at learning can predict how people will recall the words.
\end{abstract}

Keywords: Eye-tracking, memory, aging, organizational strategies, recall 
Eye-tracking and organizational strategies

One of the main hypotheses that was proposed to explain the age-related impairment of episodic memory states that older adults have trouble selecting and using efficient strategies during encoding and/or retrieval (for reviews, see Craik \& Rose, 2012; Froger, Toczé, \& Taconnat, 2014; Shing, Werkle-Bergner, Brehmer, Muller, Li, \& Lindenberger, 2010). The various strategies that people use to memorize verbal or pictorial material have been largely explored (for reviews, see Kirchhoff, 2009; Shing et al., 2010; Shing, Werkle-Bergner, Li, \& Lindenberger, 2008; Taconnat \& Lemaire, 2014). However, the nature and time course of the encoding processes are often difficult to grasp because they are mostly assessed through indirect measures. Individuals are asked to report the strategies that they used during learning, or these encoding strategies are deduced from detailed examination of the memory performance. To get a more precise picture of memorizing processes, some researchers used online measures such as eye-tracking (Loftus, 1972; Tversky, 1974; for reviews, see Foulsham \& Kingstone, 2013; Kent \& Lamberts, 2008; Pazzalia, Staub, \& Rotello, 2014). Surprisingly, most of the eye-tracking studies that explored people's encoding processes used pictorial material such as visual scenes (e.g., Sharot, Davidson, Carson, \& Phelps, 2008; Van der Linde, Rajashekar, Bovik, \& Cormack, 2009), pictures (e.g., Kafkas \& Montaldi, 2011; Molitor, Ko, Hussey, \& Ally, 2014) or faces (e.g., Henderson, Williams, \& Falk, 2005). Only very few studies were devoted to the memorization of verbal material (Cattaneo, Rosen, Vecchi, \& Pelz, 2008; Geiselman \& Bellezza, 1977; Geiselman, Woodward, \& Beatty, 1982; Pazzaglia et al., 2014; Tversky, 1974).

Hence, the present study focused on younger and older adults' encoding strategies for verbal material. As reviewed by Froger, Bouazzaoui, Isingrini, and Taconnat (2012) and Froger et al. (2014), basic monitoring of learning is largely spared in aging. However, many older adults fail to produce the effective encoding strategies adopted spontaneously by younger adults. In other words, they do not spontaneously use the processes that assist 
Eye-tracking and organizational strategies

memorization, even though they can do so when given appropriate instructions. The main aim of this study was therefore to use eye-tracking to better understand how younger and older adults spontaneously memorized sets of words. More precisely, the experiment was designed to assess whether younger and older adults used semantic and/or subjective organizational strategies when encoding words, and whether they were able to adapt their eye movement patterns to the particular features of the verbal material.

\section{Impact of aging on the use of organizational strategies in word learning}

Organizational processes are among the most efficient means to learn and recall words when no cues are provided to facilitate retrieval. One of the main organizational strategies was first described by Bousfield (1953), who showed that when presented with a randomlyordered list of words belonging to several categories (i.e., an "organizable" list of words), individuals tended to recall the words in categorical clusters, i.e., to recall consecutively the words pertaining to the same semantic category. Many experiments replicated this result and showed that people who spontaneously grouped the semantically related words together during recall could retrieve more words (Denney, 1974; Taconnat, Raz, Toczé, Bouazzaoui, Sauzéon, Fay, \& Isingrini, 2009).

When the words to learn are not semantically related, people can use another organizational process, i.e., subjective organization. People who use this strategy recall the same groups of unrelated words consecutively across successive trials, binding these words together by a subjective link in their episodic memory (Tulving, 1962; Sternberg \& Tulving, 1977; Howard \& Kahana, 1999). Numerous studies demonstrated that using a subjective organizational strategy boosted the recall performance (for a review, see Davis, Klebe, Guinther, Schroder, Cornwell, \& James, 2013; Tulving, 1962). 
Eye-tracking and organizational strategies

Many authors showed that older adults do not spontaneously use subjective organizational processes as much as younger adults when learning and recalling words (Davis et al., 2013; Hultsch, 1974; Kausler, 1994; Light, 1991; Sauzéon, Claverie \& N’Kaoua, 2006; Stuss, Craik, Sayer, Franchi, \& Alexander, 1996; Witte, Freund, \& Sebby, 1990; Witte, Freund, \& Brownwhistler 1993). In contrast, there is no consensus regarding the impact of aging on semantic organizational strategies. Some studies reported that older adults can still use semantic organization spontaneously to boost their recall performance (e.g., Kahana \& Wingfield, 2000; Kuhlmann \& Touron, 2016; Park, Smith, Dudley, \& Lafronza, 1989; Rankin, Karol, \& Tuten, 1984; Sauzéon et al., 2006). However, other authors found a significant age-related impairment of this ability (e.g., Denney, 1974; Howard, McAndrews, \& Lagasa, 1981; Taconnat et al., 2009; West \& Thorn, 2001; Zivian \& Darjes, 1983). Altogether, the ability to use semantic associations would be relatively spared in older adults compared to their ability to use subjective organization (Golomb, Peelle, Addis, Kahana, \& Wingfield, 2008; Wingfield \& Kahana, 2002). The discrepancy between the impacts of aging on these two organizational strategies suggests that these strategies rely on distinct encoding and/or retrieval processes (Howard \& Kahana, 2002), and may involve different word scanning strategies at encoding.

\section{Eye movements during encoding processes}

The experiments in which eye-tracking was used to follow the encoding of pictorial material showed that eye movement measures were linked with the later performance in item recognition or recall. In particular, the number of fixations made on a picture, an object or a face during the encoding phase was a good predictor of the strength of the memory for that item (Heisz, Pottruff \& Shore, 2013; Kafkas \& Montaldi, 2011; Loftus, 1972; Pertzov, 
Eye-tracking and organizational strategies

Avidan \& Zohary, 2009; Saint-Aubin, Tremblay, \& Jalbert, 2007; Van der Linde et al., 2009). Numerous fixations on an object or a visual scene may allow accumulation of more detailed visual information, which would be critical for the creation of a strong memory (Hollingworth \& Henderson, 2002; Kafkas \& Montaldi, 2011; Pertzov et al., 2009).

Similar results were obtained by the few authors who used eye-tracking to examine word memorization. Tversky (1974) was the first one to show that more fixations on words at encoding were associated with better word recall. Geiselman and Bellezza (1977) found that when people memorized lists of words, the number of fixations made on each word, rather than their average duration, predicted the immediate recall performance. However, the "delayed" recall performance (after 15 seconds of a distracting task) was more related to the total duration of all the fixations made on this word. In a later study, Geiselman et al. (1982) showed in addition that under delayed recall conditions, younger adults spontaneously increased the average duration of their fixations on words compared to immediate recall conditions, which suggests that they were able to adapt their word scanning strategy to the particular features of the memory task. Recently, Pazzaglia et al. (2014) found again a positive relationship between the total time a word was fixated and the word recognition performance, whereas the average gaze duration or first fixation duration on the word did not significantly predict word recognition. Refixations of the same word may actually function as supplementary presentations of the material to be learnt, resulting in the strengthening of that word in memory. Interestingly, Cattaneo et al. (2008), who looked at the way people memorized the location of images or words in a display, found that in contrast with the above data, the average duration of single gazes on a particular item was more critical than the number of gazes on that item in predicting the accuracy of its localization at test.

More generally, the relationship between eye movements and memory may be explained by the way people focus their attention while scanning scenes, pictures or words, 
Eye-tracking and organizational strategies

and by the amount of information they extract to optimize their representations in memory (Hollingworth \& Henderson, 2002; Kafkas \& Montaldi, 2011; Ryan, Leung, Turk-Browne, \& Hasher, 2007; Van der Linden et al., 2009). During the exploration of scenes or faces, eye fixations are directed toward the most salient or task-relevant features, revealing the use of feature- and context-dependent exploration strategies (for recent reviews, see Rayner, 2009; Wu, Wick, \& Pomplun, 2014). For instance, eye movement patterns can reveal how distant elements of a scene are bound together and associated in memory, and how online representations of visual scenes are built-up across viewing (e.g., Ryan et al., 2007; Ryan \& Cohen, 2004; Ryan \& Villate, 2009). In addition, both visual perception and visual search are influenced by the high-level features of objects such as their meaning and their semantic relationships (for recent reviews, see Wu et al., 2014; Yee, Overton, \& Thompson-Schill, 2009). Hwang, Wang, and Pomplun (2011) measured the eye movements of participants who were asked to memorize visual scenes in order to be able to decide afterwards if a particular object was present in the previous scene. The results revealed a preference for looking at objects that were semantically related to the currently inspected one. When searching for a particular object in each scene, participants' eye movements were preferentially guided toward the objects that were semantically related to the target. This spontaneous grouping of semantically similar objects may correspond to a semantic organizational strategy, which would allow people to memorize more details of the scene.

Only few studies examined the impact of aging on the relationship between eye movements at encoding and memory (Açik, Sarwary, Schultze-Kraft, Onat, \& König, 2010; Firestone, Turk-Browne, \& Ryan, 2007; Shih, Meadmore, \& Liversedge, 2012a, 2012b; Williams, Zacks, \& Henderson, 2009). All studies showed that older adults were impaired on memory tests compared to younger adults, but the eye-tracking data were not fully consistent. Açik et al. (2010) and Firestone et al. (2007) demonstrated that when asked to scan images or 
Eye-tracking and organizational strategies

faces for a fixed amount of time, older adults make more fixations and lower amplitude saccades than younger adults. As a result, the average duration of older adults' fixations was shorter than that of younger adults. In contrast, Shih et al. (2012a, 2012b) found that when asked to memorize a set of objects presented for ten seconds, older adults made fewer, longerlasting fixations on the objects than younger adults. The number of fixations and the total time spent on a given object predicted recognition accuracy for both younger and older adults. Similarly, Williams et al. (2009) demonstrated that during a visual search task, older adults made longer fixations on the target and distractor items than younger adults, and again the total time spent on each item predicted recognition accuracy for both groups of participants. In the two latter studies, similar results were obtained when gazes (or visits), defined as the sum of successive fixations on the same object, were considered instead of single fixations. According to Açik et al. (2010), the apparent discrepancy between the two sets of studies may result from the different viewing tasks that were used. In the two first experiments indeed (Açik et al., 2010; Firestone et al., 2007) participants had no precise target information in memory while viewing the stimuli, which means that the participants were free to explore any part of the image. In contrast, participants in the three other studies were searching for or trying to memorize well-defined target objects, and were thus able to concentrate on particular items in the scene.

\section{The current study}

As stated above, the main goal of this study was to use eye-tracking to assess whether younger and older adults differently encoded words. All participants were asked to memorize a semantically organizable set of words belonging to several categories and a non-organizable set of words to give them the opportunity to use both the semantic and/or subjective 
Eye-tracking and organizational strategies

organizational strategies. As reported in the literature, younger adults should recall more words than older adults, and all participants should recall more words from the organizable set of words than from the non-organizable one (Bäckman, \& Wahlin, 1995; Herlitz \& Viitanen, 1991, Sauzéon et al., 2006; Stuss et al., 1996). When recalling words, older adults should not spontaneously use subjective organizational processes as much as younger adults (Davis et al., 2013), but aging should have only relatively minor effects on the semantic organizational processes (Golomb et al., 2008).

Regarding the eye-tracking data, the scarcity of studies performed during word memorization makes it difficult to issue precise hypotheses. Nevertheless, in view of the existing literature, whether or not a word will be recalled by both younger and older adults should depend on the number of gazes and/or the total amount of time spent on that word during the encoding phase, at least for the non-organizable words (Pazzaglia et al., 2014; Shih et al., 2012b). In addition, since in the present study participants were trying to memorize well defined items, the average duration of older adults' gazes may be longer than that of younger adults (Açik et al., 2010; Shih et al., 2012a, 2012b).

Finally, younger adults were expected to show signs of semantic and subjective organizational processes when encoding words, and to be able to adapt their scanning pattern according to the features of each set of words. As pointed out above (Hwang et al., 2011; Wu et al., 2014; Yee et al., 2009), eye movement patterns can reveal how distant elements of a display are bound together in memory. In particular, the order in which objects are scanned suggests when people spontaneously bind together semantically-related items by looking at them in succession, and thus use semantic organizational strategies to optimize learning. As reported below, both the younger and older participants in the present study had 100 seconds to memorize 20 words, and could thus fixate each word in average about ten times. This gave them the possibility to notice that the organizable set included several categories of 
Eye-tracking and organizational strategies

semantically-related words, and to implement a semantic organizational strategy consisting in looking consecutively at the words pertaining to the same semantic category. They had also ample time to scan several times the same groups of non-organizable words consecutively in order to bind these words together by a subjective link in their episodic memory and use subjective organizational strategies. However, since as stated above older adults have trouble selecting efficient strategies during encoding (Craik \& Rose, 2012; Froger et al., 2014; Shing et al., 2010), they may not be able to adapt their encoding strategy according to the features of each set of words as well as younger adults, in particular when the best choice is to use subjective organizational processes (see Davis et al., 2013).

\section{Methods}

\section{Participants}

Two groups of participants were involved in this experiment. The 39 younger adults (19 women) were 20 to 39 years old, whereas the 50 older adults (34 women) were 60 to 91 years old. The characteristics of each group of participants are presented in Table 1. All participants were native French speakers and had normal or corrected-to-normal vision. They were volunteers and signed a written, informed consent. The older participants lived at home and were recruited from leisure clubs or from "Senior Citizens' University". The younger participants were recruited from leisure clubs and through referrals by the older participants. Participants were individually interviewed to exclude those with a history of alcoholism, undergoing treatment for psychiatric illness, or taking psychoactive medication.

The two groups were matched on their anxiety and depression scores assessed by the HADS (Hospital Anxiety and Depression Scale, Zigmond \& Snaith, 1983). These variables 
Eye-tracking and organizational strategies

were controlled because the level of anxiety may affect learning strategies, while depression, which is generally linked to self-evaluated health, is known to affect motivation in learning. Verbal abilities, which may have an impact on verbal learning, were assessed with the MillHill vocabulary test (Raven, Court, \& Raven, 1986). As shown in Table 1, older adults performed better than younger ones in this task, despite their slightly lower number of years of formal education. Hence, the lower performance of older adults in memory tasks cannot be due to lower verbal aptitudes. Older adults were also screened using the Mini Mental State Examination (MMSE, Folstein, Folstein, \& McHugh, 1975). To minimize the risk of inclusion of people suffering from preclinical dementia, the MMSE cut-off was set at 27 points. Ethical approval for the research was obtained from the Psychology Department of the University of Tours. After the experiment, participants were debriefed about the general goal of the research.

[Please insert Table 1 about here]

\section{Material}

The material consisted of two sets of 20 concrete nouns, which were all 4 to 8 letters long and included 1 to 3 syllables (see Annex 1). The first one included 20 "semantically organizable" words belonging to five different semantic categories (i.e., animals, body parts, clothing, vegetables, and vehicles), and was used to assess the participants' recall performance for organizable words and the extent to which semantic organization was used during encoding and recall. The words of the five categories were selected from Marchal and Nicolas's category association norms (2003), which were collected from both young and elderly adult French speakers. Words in each category were matched according to their 
Eye-tracking and organizational strategies

length, lexical frequency (Brulex database; Content, Mousty, \& Radeau, 1990), and frequency of production by adults in their semantic category (Marchal \& Nicolas, 2003). Their mean lexical frequency was 19.2 per million.

The second set of words included 20 "non-organizable" words (Annex 1) and was used to assess the participants' recall performance for non-organizable words and the extent to which they used subjective organization processes during encoding and recall. The words were matched with those of the organizable set with respect to length, number of syllables, concreteness, and lexical frequency.

\section{Apparatus and procedure}

The experiment was performed using a TOBII 1750 eye-tracker, which displayed the stimuli on a 17 -inch monitor (resolution $768 \times 1,024$ pixels). The eye-tracker was controlled via a Fujitsu/Siemens laptop and measured gaze positions at $50 \mathrm{~Hz}$ and with a spatial precision of about $.5^{\circ}$ of visual angle. The participants sat with their heads unsupported in front of the eye-tracker screen in a natural and relaxed position. The screen was set at the viewing distance that optimised the quality of each participant's eye movement recordings. The distance ranged from 510 to $790 \mathrm{~mm}$ depending on participants $(M=645 \mathrm{~mm}, S D=75)$, with the result that $10 \mathrm{~mm}$ on the screen covered about $.95^{\circ}$ of visual angle.

After completing the anxiety and depression scales and carrying out the verbal ability tests, all participants were tested individually with both the organizable and the nonorganizable sets of words. Half of the participants in each group began with the organizable set, whereas the other half began with the non-organizable one. Participants studied the first set of words and were then asked to recall the words of that set three times as detailed below. 
Eye-tracking and organizational strategies

Then, they studied the second set of words and were asked to recall the words of this second set. Participants could relax for a few minutes between the two tests.

Before the presentation of each set of words, the eye-tracker was calibrated using a 9point calibration procedure based on fixation stimuli distributed across the entire screen. Participants were then instructed to memorize as many words as possible of the words that were going to appear on the eye-tracker screen. They were first asked to fixate a central fixation cross. Then, the 20 words were displayed simultaneously for 100 seconds on the eyetracker screen in a way that maximised the distance between words, while avoiding horizontal or vertical alignments as far as possible (Figure 1). In the case of organizable words, care was taken to separate the words that belonged to the same category, and participants ignored beforehand that the words were semantically organizable. To avoid any impact of the spatial distribution of words, five different versions of each display were used throughout the experiment. Participants' eye movements were recorded while they memorized the words that were displayed on the screen.

[Please insert Figure 1 about here]

To avoid any recency effect, participants were asked to perform an interfering task (letter comparison task) for 45 seconds following the presentation of the words. Then, the participants were asked to recall and say aloud as many words of the set as possible. Participants were asked to recall the words three times without seeing them again to evaluate the evolution of their memory and of the underlying organizing processes. They were asked to perform the interfering task again between each trial.

\section{Measures and data analysis}


Eye-tracking and organizational strategies

Memory and organization of recall. The number of words from the organizable and non-organizable sets of words that were recalled on each trial was used to measure participants' memory performance. The recall performance was analyzed using a three-way ANOVA with age as a between-participant factor, and the set of words (organizable or nonorganizable) and rank of trials (first, second or third) as within-participant factors.

For the semantically organizable words, the semantic clustering of the words at retrieval was measured from the order in which the words were recalled using the "list-based semantic clustering index" (LBSCI) developed by Stricker, Brown, Wixted, Baldo, and Delis (2002). As discussed by Kuhlmann \& Touron (2016), list-based clustering measures would be more representative of clustering success than output-based clustering measures such as the “adjusted ratio of clustering” (Roenker, Thompson, \& Brown, 1971). Indeed, the LBSCI does not only consider the number of semantic category repetitions in the recall output, but also the number and size of the clusters relative to the number and size of categories on the original list (see also Taconnat et al., 2009; Yu, McCall, Homayouni, Tang, Chen, Schoff, et al., 2018). A LBSCI of 0 indicates chance clustering while positive values indicate significant semantic clustering.

For the non-organizable words, the consistency in the order in which the words were recalled on different trials (i.e., subjective organization) was measured using the "pairwise frequency index" (PFI, Anderson \& Watts, 1969; Sternberg \& Tulving, 1977), which assesses the proportion of word pairs recalled together across trials. The higher the score, the higher the subjective organization of recall. Because the PFI is based on the pairs of words recalled together on successive trials, only two PFI could be calculated: one between the first and second trials, and one between the second and third trials. 
Eye-tracking and organizational strategies

Both the LBSCI (for organisable words) and PFI (for non-organizable words) were analyzed using two-way ANOVAs with age as a between-participant factor and the rank of trials (first, second or third) as a within-participant factor.

Eye movement recordings. ClearView 2.7.1 software was used to record participants' eye movements on words during the encoding phase and to time slide presentations. Eye fixations were defined as any period during which the gaze stopped for 50 ms (three successive gaze points at $50 \mathrm{~Hz}$ ) or more within a 30 -pixel (about $.95^{\circ}$ of visual angle) diameter area. During encoding, a fixation was assigned to a word when it was located within $10 \mathrm{~mm}$ of the word, i.e. the distance covered by the participant's fovea. The few fixations that were located within $10 \mathrm{~mm}$ of two words were assigned to the closest one. Fixations that were not directed within $10 \mathrm{~mm}$ of any word were not assigned and were removed from the analysis. Successive fixations on the same word were collapsed together as a single gaze on the word. In contrast, re-fixations of the same word after at least one fixation elsewhere in the display were counted as a second gaze on the word.

Eye movements were first analyzed using as dependent variables the total time that participants spent gazing at the words in the display, the average number of gazes made at these words, and their average duration. The data were analyzed using two-way ANOVAs with age as a between-participants factor, and the set of words as a within-participants factor.

Organization of encoding. As stated in the introduction, the order in which objects are scanned can reveal how distant elements of a display are bound together and associated in memory. To assess whether participants used semantic organization when encoding the organizable words, and/or subjective organization when encoding the non-organizable words, the sequence of successive gazes made on the words of each set by each participant was 
Eye-tracking and organizational strategies

divided in four "quartiles". Each quartile included one fourth of the participant's total number of gazes on words.

For the organizable words, semantic organization during encoding was evaluated using an eye movement-based version of the LBSCI. The idea was to use eye-tracking to evaluate the participants' tendency to gaze consecutively at the words of the same semantic category while encoding the words. This "list-based semantic clustering index at encoding" (LBSCI-Enc) was computed separately for each quartile of the sequence of gazes to check whether the use of semantic organizing processes evolved over time while participants scanned the display. The LBSCI-Enc for a given quartile was defined using the number of semantic category repetitions $(R)$ observed in the sequence of gazes (i.e., the number of times an item from a category was gazed at just after an item from the same category) and the number of gazes made on words during the quartile $\left(f_{\text {enc }}\right)$. The "list-based semantic clustering expectancy at encoding" $\left(E X P_{\text {sem-enc }}\right)$ for a given quartile was defined as $E X P_{\text {sem-enc }}=\left(\left(f_{\text {enc }}-1\right)\right.$ $(m-1)) /\left(N_{\mathrm{L}}-1\right)$, where $m$ is the number of members of each semantic category in the set of words, and $N_{\mathrm{L}}$ is the total number of words in the set. In the present study, the formula simplifies to $\operatorname{EXP}_{\text {sem-enc }}=\left(\left(f_{\mathrm{enc}}-1\right)(4-1)\right) /(20-1)=\left(f_{\mathrm{enc}}-1\right) / 6.33$. The LBSCI-Enc was then calculated by subtracting $E X P_{\text {sem-enc }}$ from $R$ (see Stricker et al., 2002). As for the LBSCI, a LBSCI-Enc of 0 indicates chance clustering while positive values indicate significant semantic clustering.

For the non-organizable words, the participants' use of subjective organizing processes during encoding was evaluated by computing a new eye movement-based index, the pair similarity ratio (PSR). This index, which was calculated separately for each quartile of the sequence of gazes, was used to evaluate the consistency in the order in which the words were encoded (subjective organization) during the presentation of non-organizable words. The PSR for a given quartile was defined as the ratio between two measures. The first one was the 
Eye-tracking and organizational strategies

total number of pairs of consecutively gazed at words included in the sequence of gazes, which is actually the number of gazes in this part of the sequence minus one (i.e., $f_{\mathrm{enc}}-1$ ). The second one was the number of pairs of consecutively gazed at words that were different from one another, without taking into account the order in which the two words were gazed at. Consider for instance the following sequence of twelve gazes made on the following words, numbered from 1 to 20: 7-1-3-6-1-3-1-3-6-1-10-1. The total number of pairs of consecutively gazed at words is 11 (i.e., 7-1, 1-3, 3-6, 6-1, 1-3, 3-1, 1-3, 3-6, 6-1, 1-10, and 10-1), but only 5 distinct pairs of words appear in this sequence (1 and 7, 1 and 3, 3 and 6,1 and 6, 1 and 10). Hence, the PSR for this sequence is $11 / 5=2.20$. Higher PSR indicate that a high proportion of words were repeatedly scanned one after the other, which suggests a more important use of subjective organizing processes during encoding.

The LBSCI-Enc (for organizable words) and PSR (for non-organizable words) were analyzed using two-way ANOVAs with age as a between-participant factor and the rank of quartiles (first, second, third or fourth) as a within-participant factor.

\section{Results}

\section{Word recall performance}

The number of words that were recalled by younger and older adults is shown on Table 2. A three-way ANOVA was performed using age, the set of words (organizable or nonorganizable) and the rank of trials (first, second or third) as independent variables. The analysis revealed main effects of age $\left(F(1,87)=17.53, p<.001, \eta_{p}{ }^{2}=.168\right)$ and of the set of words $\left(F(1,87)=134.91, p<.001, \eta_{p}{ }^{2}=.608\right)$ on the recall performance, but no significant interaction between these two factors $(F(1,87)=.03, p=.86)$. As shown on Table 2, older 
Eye-tracking and organizational strategies

participants recalled fewer words than younger participants did, and both the younger and older adults recalled more words from the organizable set than from the non-organizable one. The recall performance decreased significantly across the three trials $(F(2,174)=8.50, p<$ $\left..001, \eta_{p}{ }^{2}=.089\right)$, but there was no significant interaction between the rank of trials and the other factors $(F<2.46$ and $p>.08$ in all cases). Planned comparisons demonstrated that for both the younger and older adults, the recall performance decreased between the first and the second trial $(F(1,87)=11.87, p<.001)$, but not between the second and third trials $(F(1,87)=$ $.49, p=.49)$.

[Please insert Table 2 about here]

\section{Organization of recall}

Table 2 gives for each group of participants the LBSCI, which was used to assess the semantic clustering of recall for the organizable words, and the PFI, which was used to evaluate the subjective organization of recall for the non-organizable words.

There was a main effect of age $\left(F(1,87)=7.47, p<.01, \eta_{p}{ }^{2}=.079\right)$ and of the rank of trials $\left(F(2,174)=4.89, p<.01, \eta_{p}{ }^{2}=.053\right)$ on the LBSCI, as well as a significant interaction between the two factors $\left(F(2,174)=7.44, p<.001, \eta_{p}{ }^{2}=.079\right)$. Planned comparisons demonstrated that the LBSCI increased across trials in younger adults (second versus first trial $F(1,87)=6.31, p<.05$, third versus second trial $F(1,87)=6.31, p<.05)$, but not in older participants (second versus first trial $F(1,87)=.01, p=.94$, third versus second trial $F(1,87)=$ $.66, p=.42$ ). The LBSCI was actually higher in younger adults than in older participants on the second and third trials (first trial $F(1,87)=2.95, p=.09$, second trial $F(1,87)=6.56, p<$ .05 , third trial $F(1,87)=12.89, p<.001)$. For both younger and older adults, there was a 
Eye-tracking and organizational strategies

positive correlation between the LBSCI and the recall performance on all three trials (younger adults $.85<r<.90, p<.001$, older adults $.58<r<.76, p<.001)$. The correlation was significantly stronger for younger adults than for older adults on the first $(p<.01)$ and third trials $(\mathrm{p}<.01)$, but not on the second one $(\mathrm{p}=.20)$.

There was a main effect of age on the PFI used to evaluate the subjective organization of recall for the non-organizable words $\left(F(1,86)=9.67, p<.01, \eta_{p}{ }^{2}=.101\right)$ and a significant effect of the rank of trials $\left(F(1,86)=4.10, p<.05, \eta_{p}{ }^{2}=.046\right)$, but no significant interaction between the two factors $(F(1,86)=1.94, p=.17)$. The PFI was higher in younger adults than in older participants and among both younger and older adults, the PFI increased between the second and the third trial. In both groups of participants, there was a positive correlation between the subjective organization of recall assessed by the PFI and the recall score on both the second (younger adults $r=.47, p<.01$; older adults $r=.62, p<.001$ ) and third trials (younger adults $r=.58, p<.01$; older adults $r=.58, p<.001$ ). There was no significant difference in the strength of this correlation between younger and older adults $(p>.32$ for both trials)

\section{Eye movement data}

Validity and precision of eye movement recordings. The validity of participants' eye movement recordings was checked using the estimation provided by ClearView ${ }^{\circledR}$ software. Valid data were obtained for $M=96.3 \%(S D=3.1)$ of data points in younger adults (range $87.9-99.9 \%)$, and for $M=92.1 \%(S D=5.9)$ of data points in older participants (range $75.9-$ 99.7\%). Despite the small amplitude of this difference, the validity of eye movement data was significantly higher in younger than in older participants (Mann-Whitney $U=505.0, z=-$ $3.88, p<.001)$. This means that the total time spent gazing at words during encoding was 
Eye-tracking and organizational strategies

artificially reduced in older participants. To ensure the accuracy of comparisons between younger and older participants, the total time spent gazing at words versus other parts of the displays or of the visual environment was expressed for each participant as a proportion of the valid recordings obtained for each set of words.

The spatial precision of participants' eye movement recordings was checked by examining the fixations recorded while participants were looking at the fixation cross that was displayed before each set of words. The average distance between the cross and the participant's fixations was $M=14.7$ pixels (about $.5^{\circ}$ of visual angle, $S D=7.0$ ) in younger adults (range $3.0-29.5)$, and $M=15.7$ pixels $(S D=7.6)$ in older participants (range $2.5-$ 37.5). There was no significant difference in the precision of eye movement recordings between younger and older adults $(t(86)=.66, p=.51)$.

[Please insert Table 3 about here]

Total time spent gazing at words. Table 3 gives for each group of participants and each set of words the average proportions of time participants spent gazing at the words, gazing between the words or outside the screen, and moving the eyes between gazes.

The total proportion of time spent gazing at words was higher for younger than for older adults $\left(78 \%\right.$ versus $\left.73 \%, F(1,87)=6.62, p<.05, \eta_{p}{ }^{2}=.071\right)$, but was not significantly different between the organizable and the non-organizable words $(F(1,87)=1.95, p=.17)$. Conversely, the proportion of time spent gazing between words or outside the screen was slightly higher for older adults than for younger ones (4.5\% versus $3.5 \%, F(1,87)=4.97, p<$ $\left..05, \eta_{p}{ }^{2}=.054\right)$. In addition, the proportion of time spent gazing between words or outside the screen was higher for the organizable words than for the non-organizable ones $(4.5 \%$ versus $\left.3.5 \%, F(1,87)=13.87, p<.001, \eta_{p}{ }^{2}=.137\right)$. Finally, older adults spent also more time 
Eye-tracking and organizational strategies

moving their eyes between gazes (i.e., making saccades) than younger adults $(22.5 \%$ versus $\left.18.5 \%, F(1,87)=4.40, p<.05, \eta_{p}{ }^{2}=.048\right)$. The proportion of time spent making saccades did not differ between the organizable and non-organizable words $(F(1,87)=.51, p=.48)$.

Interestingly, the size of the saccades made while encoding the words did not significantly depend on participants' age $(F(1,87)=.91, p=.34)$. Actually, participants' average saccade size (about $5.0^{\circ}$ of visual angle) tended to be smaller for older adults than for younger adults $(M=149$ pixels, $S D=29$ versus $M=158$ pixels, $S D=37$ for the organizable words, $M=142$ pixels, $S D=35$ versus $M=147$ pixels, $S D=39$ for the non-organizable ones). Hence, older adults spent more time moving their eyes between gazes than younger adults because of a decrease in saccadic velocity. In addition, there was a main effect of the set of words on participants' average saccade size. Both older and younger adults made longer saccades when the words were organizable than when they were not organizable $(F(1,87)=$ 14.56, $\left.p<.001, \eta_{p}^{2}=.143\right)$. The interaction between age and the set of words was not significant $(F(1,87)=.74, p=.39)$.

Number and average duration of the gazes made on words. Figure 2 gives for each set of words the average number of gazes made on words and average gaze duration. For both groups of participants, neither the rate nor the average duration of gazes varied significantly over the 100 seconds study time allotted to each set of words.

[Please insert Figure 2 about here]

There were main effects of age $\left(F(1,87)=10.96, p<.01, \eta_{p}{ }^{2}=.112\right)$ and of the set of words $\left(F(1,87)=32.32, p<.001, \eta_{p}{ }^{2}=.271\right)$ on the number of gazes made on words during encoding. There was also an interaction between the two factors $\left(F(1,87)=10.49, p<.01, \eta_{p}{ }^{2}\right.$ 
Eye-tracking and organizational strategies

$=.108)$. As shown on Figure 2, younger participants made more gazes on words when encoding the organizable words than when encoding the non-organizable ones $(F(1,87)=$ $35.43, p<.001)$, whereas the difference was not significant for older adults $(F(1,87)=3.42, p$ $=.07)$. As a result, younger adults made more gazes on words than older adults when encoding the organizable words $(F(1,87)=17.62, p<.001)$, whereas the difference was not significant for the non-organizable words $(F(1,87)=3.34, p=.07)$. Interestingly, for the organizable words only, there was a positive correlation among both younger and older adults between the number of gazes made on words at encoding and the recall performance on all trials (younger adults $.42<\mathrm{r}<.49, \mathrm{p}<.01$ in all cases, older adults $.31<\mathrm{r}<.35, \mathrm{p}<.05$ ). The younger and older adults that recalled the most organizable words were those who made the highest number of gazes on each word during encoding.

Regarding average gaze durations, the analysis revealed no significant main effect of age $(F(1,87)=.02, p=.88)$, but a main effect of the set of words $(F(1,87)=16.10, p<.001$, $\left.\eta_{p}{ }^{2}=.156\right)$ and a significant interaction between the two factors $\left(F(1,87)=5.11, p<.05, \eta_{p}^{2}\right.$ $=.056)$. As shown on Figure 2, older participants' average gaze duration was not significantly different between the two sets of words $(F(1,87)=1.75, p=.19)$. In contrast, younger participants made longer gazes on the non-organizable words than on the organizable ones $(F(1,87)=17.52, p<.001)$. Whatever the set of word, there was no significant relationship between either younger or older adults' average gaze durations and their recall performance.

Measures of organization at encoding. Table 4 gives the LBSCI-Enc used to evaluate semantic clustering at encoding for the organizable words, and the PSR used subjective organization at encoding for the non-organizable words. These measures were computed separately for each quartile of the encoding phase. 
Eye-tracking and organizational strategies

[Please insert Table 4 about here]

There was no significant effect of age on the LBSCI-Enc $(F(1,87)=1.54, p=.22)$, but there was a significant variation of the LBSCI-Enc across the four quartiles $(F(3,261)=5.45$, $\left.p<.01, \eta_{p}{ }^{2}=.059\right)$. There was no significant interaction between the two factors $(F(3,261)=$ $1.12, p=.34)$. Planned comparisons demonstrated that the LBSCI-Enc was lower during the first quartile of the encoding phase than during the three following quartiles (LBSCI-Enc: $F(1,87)>4.99$ and $p<.05$ for the three comparisons). This suggests that when learning the organizable words, participants began to use semantic clustering only during the second quartile of the encoding phase. Among younger adults, there was a significant, positive relationship between the LBSCI-Enc obtained during the third and fourth quartiles of the encoding phase and the recall performance on all trials $(.32<\mathrm{r}<.41, \mathrm{p}<.05$ in all cases $)$. Among older adults in contrast, there was no significant relationship between the LBSCI-Enc and the recall performance on any trial $(-.09<\mathrm{r}<.23, \mathrm{p}>.10$ in all cases $)$. Similarly, among younger adults, there was a significant, positive relationship between the LBSCI-Enc obtained during the third and fourth quartiles of the encoding phase and the amount of semantic clustering at recall on the second and third trials $(.34<\mathrm{r}<.46, \mathrm{p}<.05$ in all cases), whereas this was not true among older adults $(.06<\mathrm{r}<.20, \mathrm{p}>.17$ in all cases).

For the PSR computed for non-organizable words (Table 4), the ANOVA revealed a main effect of age $\left(F(1,87)=13.81, p<.001, \eta_{p}{ }^{2}=.137\right)$, but no significant variation across the four quartiles $(F(3,261)=.35, p=.79)$ and no significant interaction between the two factors $(F(3,261)=1.20, p=.31)$. During encoding of non-organizable words, the level of subjective organization was higher for younger adults than for older adults. There was, however, no significant relationship between participants' PSR and either the PFI used to 
Eye-tracking and organizational strategies

evaluate the subjective organization of recall for the non-organizable words or their recall performance.

\section{Relationship between the eye movement data and the recall of each word}

To assess whether the words that were recalled by participants were gazed at for longer times than the other words during the encoding phase, the words that were recalled by each participant were identified for each one of the three retrieval trials. The total gaze time on each word was analyzed by performing a four-way ANOVA using age, the set of words, the rank of trials (first, second or third) and the recall status of each word as dependent variables (Table 5). The analysis revealed a main effect of age $\left(F(1,85)=10.91, p<.01, \eta_{p}{ }^{2}=\right.$ $.114)$, a main effect of the recall status of words $\left(F(1,85)=28.51, p<.001, \eta_{p}{ }^{2}=.251\right)$, and a significant interaction between the recall status of words and the set of words $(F(1,85)=6.32$, $\left.p<.05, \eta_{p}{ }^{2}=.069\right)$. In both the non-organizable set and the organizable set of words, the words that were recalled were gazed at for longer total times than those that were not recalled. However, the total time spent gazing at each particular word influenced much more the recall status of words in the non-organizable set $(F(1,85)=27.86, p<.001)$ than in the organizable one $(F(1,85)=6.50, p<.05)$. There was no other significant interaction between the recall status of words and the other dependent variables ( $F<1.98$ and $p>.14$ in all cases).

[Please insert Table 5 about here]

The longer total times spent gazing at the non-organizable words that were recalled were linked to an increase of the number of gazes made on these words, but not of their average durations. Indeed, there was a main effect of the recall status of words $(F(1,85)=$ 
Eye-tracking and organizational strategies

$\left.17.59, p<.001, \eta_{p}{ }^{2}=.171\right)$ on the number of gazes, and a significant interaction between the recall status of words and the set of words $\left(F(1,85)=3.96, p<.05, \eta_{p}^{2}=.045\right)$. In the nonorganizable set of words $(F(1,85)=18.60, p<.001)$, but not in the organizable set, the words that were recalled were gazed at more often than those that were not recalled. Average gaze durations, in contrast, did not significantly depend on the recall status of words $(F(1,85)=.34$, $p=.56)$, and there was no interaction between the recall status of words and the other dependent variables $(F<1.71$ and $p>.18$ in all cases).

\section{Discussion}

\section{Memory performance and organization of word recall}

As expected (see Table 2), older participants recalled fewer words than younger participants, and both younger and older adults recalled more words from the organizable set of words than from the non-organizable one (Bäckman \& Wahlin, 1995; Herlitz \& Viitanen, 1991; Sauzeon et al., 2006; Stuss et al., 1996). As expected also, the PFI indicated that older adults made less use of subjective organizational processes when recalling the nonorganizable words than younger adults (Davis et al., 2013; Hultsch, 1974; Kausler, 1994; Light, 1991; Sauzéon et al., 2006; Stuss, et al., 1996; Witte et al., 1990; 1993). Nevertheless, the positive correlation between the PFI and the recall scores obtained in both groups of participants shows that using subjective organization improved the recall performance for both younger and older adults. Note that the procedure used in the present study to assess whether younger and older adults used subjective organizational strategies when recalling words was different from that used in Tulving's (1962) study, in which the same word lists were presented multiple times in different orders and subjective organization was inferred 
Eye-tracking and organizational strategies

from the consistency in recall order. The present procedure was taken from Sauzéon and al. (2006), who compared the recall performance of younger and older adults for both semantically organizable and non organizable lists of words. Indeed, one of our aims was to compare participants' eye movement patterns on semantically organizable versus non organizable lists of words, which means that the exact same procedure had to be used for both types of list. Future research may test whether multiple presentations of the non organizable list may allow both younger and older adults to make more use of subjective organizational processes when recalling the words.

For the organizable words, both younger and older adults spontaneously used semantic clustering when recalling words. In accordance with the literature (Golomb et al., 2008; Wingfield \& Kahana, 2002), aging had a relatively low impact on the amount of semantic organization at recall. Indeed, the older adults' LBSCI was lower than that of younger adults on the second and third trials only. However, the use of semantic clustering increased across trials in younger adults only, and the beneficial impact of semantic clustering on the recall performance (i.e., the correlation between the LBSCI and recall scores) was stronger in younger than in older adults. In sum, the word recall data were in accordance with expectations.

According to Kausler (1994), older adults tend to show less-pronounced agedifferences when the words or material to recall can be meaningfully organized. In the present experiment, however, the impact of age on the word recall performance did not significantly differ between the organizable and non-organizable sets of words, in line with previous studies that compared memory for organizable and non-organizable lists of words in younger and older adults (Sauzéon et al., 2006; Stuss et al., 1996). Hence, even though the ability to use semantic associations would be relatively spared in older adults compared to their ability to use subjective organization, this would not be sufficient to reduce the age-related memory 
Eye-tracking and organizational strategies

deficit in the recall of organizable words. This fits well with the fact that, as stated above, the beneficial impact of semantic clustering on the recall performance was weaker in older adults than in the younger ones. As pointed out by Kuhlmann and Touron (2016), many other moderating factors in the task environment can either foster or hinder the efficiency of older adults' strategy use and contribute to age-group differences in episodic memory (see also Kausler, 1994).

\section{Impact of age on the number and duration of gazes made while encoding words}

Older adults do not look at the words for as long as younger adults. One obvious finding was that even when correcting for the lower validity of older adults' eye movement recordings ${ }^{1}$, older adults spent in total about five seconds less gazing at the words in the displays than younger adults (Table 3). Instead of gazing at the words, they spent slightly more time than younger adults gazing between the words or outside the screen, and above all they spent about four seconds more moving their eyes between gazes, i.e., making saccades. The latter result was not due to an increase in the size of older adults' saccades, nor to an increase in their number since older adults made fewer gazes on the display than younger adults, and can only be explained by a decrease in older adults' saccadic velocity.

This decrease probably results from the general slowing of motor processes observed in aging people (for a review, see Seidler, Bernard, Burutolu, Fling, Gordon, Gwin, et al., 2010), which provokes a substantial increase in the latency and duration of older adults' rapid eye movements (for a review, see Dowiasch, Marx, Einhauser, \& Bremmer, 2015). Indeed, the mean and peak velocity of saccades is reduced by about $20 \%$ in older adults compared to younger ones, while saccade duration increases by about 15\% (Dowiasch et al., 2015; Munoz,

\footnotetext{
${ }^{1}$ Despite the difference in recordings' validity, the precision of eye movement data was similar for both groups of participants.
} 
Eye-tracking and organizational strategies

Brouhgton, Goldring, \& Armstrong, 1998). This would be one of the main causes of the $20 \%$ increase of the time spent moving the eyes during word encoding seen in older participants.

In view of the positive relationship between the total time spent gazing at words during encoding and the word recognition performance reported in the literature (see Pazzaglia et al., 2009), the shorter total time spent looking at words by older adults may be one of the reasons for their lower memory performance. Obviously, the reduction of saccadic velocity also reflects the general degradation of physiological processes associated with brain aging (Lu, Lee, Raven, Tingus, Khoo, Thompson, \& Bartzokis, 2011), which would be the seminal cause for older adults' worse memory.

Contrary to what was observed for objects in previous studies (Shih et al., 2012a, 2012b), older adults' average gaze duration on words was not significantly longer than that of younger adults (Figure 2). The reason for this discrepancy may be that in Shih et al.'s (2012a, 2012b) studies, the set of 12 objects to memorize was presented for only ten seconds, i.e., for a much shorter time than in the present work, which means that the amount of times an object could be gazed at was very limited. In that particular situation, participants may increase gaze duration to ensure deeper processing of each item because they know they will not have much time to look at it again.

\section{Younger adults, but not older ones, adapted their gaze behavior to words} organizability. The eye movement data revealed major differences in the way younger and older adults encoded the two sets of words (Figure 2). Younger adults, but not older ones, made more gazes on words when encoding the organizable words than when encoding the non-organizable ones, which suggests that only younger adults were able to adapt their scanning strategy on spotting the semantic relationships between the words. Since in both groups of participants, the number of gazes made on organizable words at encoding was 
Eye-tracking and organizational strategies

positively correlated with the recall performance, this adaptive behavior may be one of the reasons for their better recall scores. Similarly, only younger adults gazed for longer average durations at the non-organizable words than at the organizable ones. This suggests that contrary to older adults, they were able to adapt their gaze durations to the absence of any semantic link between the words, which must have helped them obtain better recall scores.

Complementary analyses were performed to assess whether during the encoding phase, participants gazed differently at the words they later recalled than at those they did not recall (Table 5). Interestingly, the data revealed a dissociation between the organizable and non-organizable words. For the non-organizable words, both younger and older adults gazed for much longer total times at the words they recalled than at the words they did not recall. In both groups, this increase resulted from the fact that participants gazed more often, but not for longer durations, at the words they recalled. In contrast, among either younger or older adults, there was no significant relationship between the overall number or average duration of the gazes made on non-organizable words at encoding and the recall performance. For the organizable words, the reverse pattern was observed. Indeed, during the encoding phase, there was only a weak difference between the total time spent gazing at the words that were recalled and the time spent gazing at those that were not recalled. However, as already stated above, there was a positive relationship among both younger and older adults between the recall performance and the number of gazes made on the organizable words at encoding.

In sum, these data suggest that for the non-organizable words, whether or not a word will be recalled by a participant is determined at the word level and depends mostly on the time that the participant will spend gazing at this particular word, i.e. on the amount of processing time that will be devoted to this word. This would be true for both younger and older adults. For the organizable words, in contrast, whether or not a word will be later recalled does not depend as much on the time that the participant spent gazing at this 
Eye-tracking and organizational strategies

particular word. For both younger and older adults, the recall performance would rather depend on the overall characteristics of the eye movements made while encoding the words, and more precisely on the number of gazes made on each word.

\section{Organizational strategies of younger and older adults during word encoding}

The measure of semantic organization obtained from participants' eye movements during the encoding of organizable words (LBSCI-Enc) shows that from the second quartile of the presentation of the organizable set of words, the two groups of participants began to gaze consecutively at words of the same semantic category more than when the display appeared. According to the literature (Hwang et al., 2011; Ryan et al., 2007, Ryan \& Villate, 2009; Wu et al., 2014; Yee et al., 2009), the order in which words are scanned reflects how participants bind together semantically-related items by looking at them in succession. Since the average duration of participants' gazes did not vary significantly over the 100 seconds study time allotted to each set of words, this suggests that after about 25 seconds of presentation of the organizable words, both younger and older adults realized that the words they were trying to memorize could be grouped in categorical clusters, and tried to encode together the words of the same category. In other words, both younger and older adults would spontaneously use semantic organizational strategies when encoding the organizable words as they do when they recall them. However, since care was taken to sufficiently separate the words that belonged to the same category when constructing the displays of organizable words, participants may not have been able to gaze consecutively at more than two or three of these words simply because of the large size of the display and the high density of words on the screen (see Figure 1). This may explain why the LBSCI-Enc was highly variable and did generally not reach highly positive values. 
Eye-tracking and organizational strategies

Even though both younger and older adults seemed to use semantic organization when encoding the organizable words, positive relationships between the eye-tracking based measures of semantic organization and each participant's recall performance was only obtained among younger adults. While the older adults' eye movements suggest that at least some of them spontaneously tried to use the semantic organizational strategy at encoding, they seemed unable to do so in an efficient way. The binding that older adults establish between words of the same category may be too weak to persist over the distracting task up until the word-retrieval phase. Alternatively, older adults may use a word repetition strategy, either instead of the organizational strategy or on top of it (e.g., Froger et al., 2012). This additional strategy might compete with the organizational strategy, thus reducing its positive impact on memory. Whatever the reason, the absence of impact of organization at encoding in older adults mirrors the fact that older adults benefited less than younger ones from using semantic clustering during the recall phase. This suggests that older adults do not necessarily benefit from organizational strategies as much as younger adults when they use them.

For the non-organizable words, in accordance with expectations (see Davis et al., 2013), the pair similarity ratio (PSR) revealed that younger adults used more subjective organization at encoding than older adults. For both groups of participants, the PSR did not significantly evolve over time, which strongly suggests that to scan words in the same order repetitively was a kind of default behavior for both younger and older adults who, however, would not spontaneously use it to the same extent. In accordance with this idea, there was no significant correlation between the level of subjective organization of words observed at encoding and participants' recall performance.

\section{Synthesis and conclusions}


Eye-tracking and organizational strategies

Whether or not the words to memorize were organizable, older participants recalled fewer words than younger participants. The eye-tracking data suggest that one of the main reasons may simply be that when they encode the words, older adults do not look at them as much and for as long as younger adults, probably because of slower eye movements.

When recalling semantically organizable words, both younger and older adults spontaneously used semantic organizational processes, which allowed them to recall more words than when the words were not organizable. The eye-tracking data strongly suggest that both younger and older adults noticed the semantic relationships between the words while encoding them, and tried to use the semantic organizational strategy by gazing successively at the words from the same category. However, younger adults were apparently better able than older adults to adapt their encoding strategy to this particular feature. Indeed, only younger adults made more gazes on the organizable words than on the non-organizable ones, which may have allowed them to better assess and memorize the semantic relationships between the words.

Surprisingly, whether or not older adults adapted their scanning pattern in front of the organizable words or used semantic clustering when recalling the words did not have that much impact on their recall performance. In contrast, younger adults' recall performance for the organizable words was strongly correlated with the amount of semantic clustering observed at recall, and depended significantly on their level of use of semantic organization at encoding. Altogether, the low impact of the way the words were encoded and recalled by older adults on their recall performance confirms that they do not always benefit from using organizational strategies (Kuhlmann \& Touron, 2016; Taconnat et al., 2009).

When recalling the non-organizable words, older adults did not use subjective organizational processes as much as younger adults, but using this type of organization had a 
Eye-tracking and organizational strategies

positive impact on recall for both groups of participants. The eye-tracking data obtained at encoding revealed first that in contrast with what was observed for the organizable words, whether or not a word was recalled depended on the time that the participant, whether young or old, spent gazing at this particular word. Interestingly, only younger adults spontaneously gazed for longer average durations at the non-organizable words than at the organizable ones, which could partly explain why they recalled the non-organizable words better than older adults.

Regarding the use of organizational strategies during the encoding phase, the eyetracking data revealed that as during the recall phase, older adults did not use subjective organization of the words as much as younger adults. Nevertheless, whatever the age of participants, there was no significant relationship between the participants' recall performance and their amount of use of subjective organizational processes at encoding, which suggests that scanning words repetitively in the same order may be a kind of default behavior for all participants.

Altogether, two major results emerge from the present study. Firstly, this experiment shows that eye-tracking data may be good indices of the use of organizational strategies during the memorization of verbal items. Secondly, successful encoding of organizable words and non-organizable words rests on two different strategies. Multiplying the number of fixations in order to bind together the semantically-related words seems to be the best strategy to memorize the organizable words, whereas for non-organizable words, the most efficient strategy would be to increase the total time spent looking at each word. In addition, the data reveal that the way in which words are gazed at during encoding can predict subsequent performance in word recall.

\section{Acknowledgements}


Eye-tracking and organizational strategies

The authors thank Jean Pylouster for his excellent technical assistance in the analysis of the eye movement data and all the participants for their time and cooperation.

\section{References}

Açık, A., Sarwary, A., Schultze-Kraft, R., Onat, S., \& König, P. (2010). Developmental changes in natural viewing behavior: bottom-up and top-down differences between children, young adults and older adults. Frontiers in Psychology, 1, article 207. doi: 10.3389/fpsyg.2010.00207

Anderson, R. C., \& Watts, G. H. (1969). Bidirectional associations in multi-trial free recall. Psychonomic Science, 15, 288-289. http://dx.doi.org/10.3758/BF03336303

Bäckman, L., \& Wahlin, Å. (1995) Influences of item organizability and semantic retrieval cues on word recall in very old age. Aging \& Cognition, 2, 312-325. http://dx.doi.org/10.1080/13825589508256606

Bousfield, W. A. (1953). The Occurrence of Clustering in the Recall of Randomly Arranged Associates. The Journal of General Psychology, 49, 229-240. http://dx.doi.org/10.1080/00221309.1953.9710088

Cattaneo, Z., Rosen, M., Vecchi, T., \& Pelz, J. B. (2008). Monitoring eye movements to investigate the picture superiority effect in spatial memory. Perception, 37, 34-49. DOI: $10.1068 / \mathrm{p} 5623$ 
Eye-tracking and organizational strategies

Content, A., Mousty, P., \& Radeau, M. (1990). Brulex - A computerized database for the French language. Année Psychologique, 90, 551-566. DOI : https://doi.org/10.3406/psy.1990.29428

Craik, F. I. M., \& Rose, N. S. (2012). Memory encoding and aging: A neurocognitive perspective. Neuroscience and Biobehavioral Reviews, 36, 1729-1739. DOI: 10.1016/j.neubiorev.2011.11.007

Davis, H. P., Klebe, K. J., Guinther, P. M., Schroder, K. B., Cornwell, R. E., \& James, L. E. (2013). Subjective Organization, Verbal Learning, and Forgetting Across the Life Span: From 5 to 89. Experimental Aging Research, 39, 1-26. doi: 10.1080/0361073X.2013.741956

Denney, N. W. (1974). Clustering in middle and old age. Developmental Psychology, 10, 471475. http://dx.doi.org/10.1037/h0036604

Dowiasch, S., Marx, S., Einhauser, W., \& Bremmer, F. (2015). Effects of aging on eye movements in the real world. Frontiers in Human Neuroscience, 9, article 46. doi: 10.3389/fnhum.2015.00046

Firestone, A., Turk-Browne, N. B., \& Ryan, J. D. (2007). Age-related deficits in face recognition are related to underlying changes in scanning behavior. Aging, Neuropsychology, and Cognition, 14, 594-607. DOI: 10.1080/13825580600899717 
Eye-tracking and organizational strategies

Folstein, M. F., Folstein, S. E., \& McHugh, P. R. (1975). Mini Cognitive State: A practical method for grading the cognitive state of patients for the clinician. Journal of Psychiatric Research, 12, 189-198.

Foulsham, T. \& Kingstone, A. (2013). Fixation-Dependent Memory for Natural Scenes: An Experimental Test of Scanpath Theory. Journal of Experimental Psychology: General, 142, 41-56. doi: 10.1037/a0028227

Froger, C., Bouazzaoui, B., Isingrini, M., \& Taconnat, L. (2012). Study Time Allocation Deficit of Older Adults: The Role of Environmental Support at Encoding? Psychology and Aging, 27, 577-588. http://dx.doi.org/10.1037/a0026358

Froger, C., Toczé, C., \& Taconnat, L. (2014). How the strategic behavior modification can explain the age-related deficit in episodic memory. Année Psychologique, 114, 355-387. http://dx.doi.org/10.4074/S0003503314002061

Geiselman, R. E., \& Bellezza, F. S. (1977). Eye movements and overt rehearsal in word recall. Journal of Experimental Psychology: Human Learning and Memory, 3, 305-315. http://dx.doi.org/10.1037/0278-7393.3.3.305

Geiselman, R. E., Woodward, J. A., \& Beatty, J. (1982). Individual differences in verbal memory performance: A test of alternative information-processing models. Journal of Experimental Psychology: General, 111, 109-134. http://dx.doi.org/10.1037/00963445.111.1.109 
Eye-tracking and organizational strategies

Golomb, J. D., Peelle, J. E., Addis, K. M., Kahana, M. J., \& Wingfield, A. (2008). Effects of adult aging on utilization of temporal and semantic associations during free and serial recall. Memory \& Cognition, 36, 947-956. https://doi.org/10.3758/MC.36.5.947

Heisz, J. J., Pottruff M. M., \& Shore, D. I. (2013). Females scan more than males: A potential mechanism for sex differences in recognition memory. Psychological Science, 24, 1157-1163. DOI: $10.1177 / 0956797612468281$

Henderson, J. M., Williams, C. C., \& Falk, R. J. (2005). Eye movements are functional during face learning. Memory \& Cognition, 33, 98-106. https://doi.org/10.3758/BF03195300

Herlitz, A. \& Viitanen, M. (1991). Semantic organization and verbal episodic memory in patients with mild and moderate Alzheimer's disease. Journal of Clinical and Experimental Neuropsychology, 13, 559-574. https://doi.org/10.1080/01688639108401071

Hollingworth, A., \& Henderson, J. M. (2002). Accurate visual memory for previously attended objects in natural scenes. Journal of Experimental Psychology: Human Perception and Performance, 28, 113-136. http://dx.doi.org/10.1037/0096-1523.28.1.113

Howard, M. W., \& Kahana, M. J. (1999). Contextual variability and serial position effects in free recall. Journal of Experimental Psychology: Learning, Memory and Cognition, 25, 923941. http://dx.doi.org/10.1037/0278-7393.25.4.923 
Eye-tracking and organizational strategies

Howard, M. W., \& Kahana, M. J. (2002). When Does Semantic Similarity Help Episodic Retrieval? Journal of Memory and Language, 46, 85-98. http://dx.doi.org/10.1006/jmla.2001.2798

Howard, D. V., McAndrews, M. P., \& Lagasa, M. I. (1981). Semantic priming of lexical decisions in young and old adults. Journal of Gerontology, 36, 707-714.

Hultsch, D. F. (1974). Learning to learn in adulthood. Journal of Gerontology, 29, 302-308. http://dx.doi.org/10.1093/geronj/29.3.302

Hwang, A. D., Wang, H.-C., \& Pomplun, M. (2011). Semantic guidance of eye movements in real-world scenes. Vision Research, 51, 1192-1205. doi: 10.1016/j.visres.2011.03.010

Kafkas, A., \& Montaldi, D. (2011). Recognition memory strength is predicted by pupillary responses at encoding while fixation patterns distinguish recollection from familiarity. The Quarterly Journal of Experimental Psychology, 64, 1971-1989. doi: $10.1080 / 17470218.2011 .588335$

Kahana, M. J., \& Wingfield, A. (2000). A functional relation between learning and organization in free recall. Psychonomic Bulletin \& Review, 7, 516-521. https://doi.org/10.3758/BF03214365

Kausler, D. H. (1994). Learning and Memory in Normal Aging. San Diego, CA: Academic Press. 
Eye-tracking and organizational strategies

Kent, C., \& Lamberts, K. (2008). The encoding-retrieval relationship: retrieval as mental $\begin{array}{lllll}\text { simulation. } & \text { Trends } & \text { in } & \text { Cognitive }\end{array}$ http://dx.doi.org/10.1016/j.tics.2007.12.004

Kirchhoff, B. A. (2009). Individual Differences in Episodic Memory: The Role of Self$\begin{array}{lllll}\text { initiated } & \text { Encoding } & \text { Strategies. } & \text { Neuroscientist, } & 15,\end{array}$ http://dx.doi.org/10.1177/1073858408329507

Kuhlmann, B. G., \& Touron, D. R. (2016). Aging and Memory Improvement Through Semantic Clustering: The Role of List-Presentation Format. Psychology and Aging, 31, 771785. http://dx.doi.org/10.1037/pag0000117

Light, L. L. (1991). Memory and aging - Four hypotheses in search of data. Annual Review of Psychology, 42, 333-376. https://doi.org/10.1146/annurev.ps.42.020191.002001

Loftus, G. R. (1972). Eye fixations and recognition memory for pictures. Cognitive Psychology, 3, 525-551. http://dx.doi.org/10.1016/0010-0285(72)90021-7

Lu, P. H., Lee, G. J., Raven, E. P., Tingus, K., Khoo, T., Thompson, P. M., \& Bartzokis, G. (2011). Age-related slowing in cognitive processing speed is associated with myelin integrity in a very healthy elderly sample. Journal of Clinical and Experimental Neuropsychology, 33(10), 1059-1068. doi:10.1080/13803395.2011.59539 
Eye-tracking and organizational strategies

Marchal, A., \& Nicolas, S. (2003). Category generation norms for 38 semantic categories in young and older subjects. Année Psychologique, 103, 313-366. DOI : https://doi.org/10.3406/psy.2003.29639

Molitor, R. J., Ko, P. C., Hussey, E. P., \& Ally, B. A. (2014). Memory-related eye movements challenge behavioral measures of pattern completion and pattern separation. Hippocampus, 24, 666-672. DOI: 10.1002/hipo.22256

Munoz, D. P., Broughton, J. R., Goldring, J. E., \& Armstrong, I. T. (1998). Age-related performance of human subjects on saccadic eye movement tasks. Experimental Brain Research, 121, 391-400. DOI: 10.1007/s002210050473

Park, D. C., Smith, A. D., Dudley, W. N., \& Lafronza, V. N. (1989). Effects of age and a divided attention task presented during encoding and retrieval on memory. Journal of Experimental Psychology: Learning, Memory and Cognition, 15, 1185-1191. http://dx.doi.org/10.1037/0278-7393.15.6.1185

Pazzaglia, A. M., Staub, A., \& Rotello, C. M. (2014). Encoding time and the mirror effect in recognition memory: Evidence from eyetracking. Journal of Memory and Language, 75, 7792. http://dx.doi.org/10.1016/j.jml.2014.05.009

Pertzov, Y., Avidan, G., \& Zohary, E. (2009). Accumulation of visual information across multiple fixations. Journal of Vision, 9:2, 1-12. DOI: 10.1167/9.10.2 
Eye-tracking and organizational strategies

Rankin, J. L., Karol, R., \& Tuten, C. (1984). Strategy use, recall, and recall organization in young, middle-aged, and elderly adults. Experimental Aging Research, 10, 193-196. http://dx.doi.org/10.1080/03610738408258463

Raven, J. C., Court, J. H., \& Raven, J. (1986). Manual for Raven's progressive matrices and vocabulary scales. Oxford, England: Oxford Psychologists Press.

Rayner, K. (2009). Eye movements and attention in reading, scene perception, and visual search. The Quarterly Journal of Experimental Psychology, 62, 1457-1506. https://doi.org/10.1080/17470210902816461

Roenker, D. L., Thompson, C. P., \& Brown, S. C. (1971). Comparison of measures for the estimation of clustering in free recall. Psychological Bulletin, 76, 45-48. http://dx.doi.org/10.1037/h0031355

Ryan, J. D., \& Cohen, N. J. (2004). The nature of change detection and online representations of scenes. Journal of Experimental Psychology: Human Perception and Performance, 30, 988-1015. http://dx.doi.org/10.1037/0096-1523.30.5.988

Ryan, J. D., Leung, G., Turk-Browne, N. B., \& Hasher, L. (2007). Assessment of age-related changes in inhibition and binding using eye movement monitoring. Psychology and Aging, 22, 239-250. DOI: 10.1037/0882-7974.22.2.239 
Eye-tracking and organizational strategies

Ryan, J. D., \& Villate, C. (2009). Building visual representations: The binding of relative spatial relations across time. Visual Cognition, 17, 254-272. DOI: $10.1080 / 13506280802336362$

Saint-Aubin, J., Tremblay, S., \& Jalbert, A. (2007). Eye movements and serial memory for visual-spatial information. Does time spent fixating contribute to recall? Experimental Psychology, 54, 264-272. http://dx.doi.org/10.1027/1618-3169.54.4.264

Sauzéon, H., Claverie, B., \& N'Kaoua, B. (2006). Age Differences in the Organization and Acquisition-Forgetting Processes in a Multi-Free-Recall Task. Current Psychology Letters [Online], 18, http://journals.openedition.org/cpl/1012.

Seidler, R. D., Bernard, J. A., Burutolu, T. B., Fling, B. W., Gordon, M. T., Gwin, J. T., Kwak, Y., \& Lipps, D. B. (2010). Motor control and aging: Links to age-related brain structural, functional, and biochemical effects. Neuroscience \& Biobehavioral Reviews, 34, 721-733. DOI: 10.1016/j.neubiorev.2009.10.005

Sharot, T., Davidson, M. L., Carson, M. M., \& Phelps, E. A. (2008). Eye Movements Predict Recollective Experience. PLoS One, 3, e2884. https://doi.org/10.1371/journal.pone.0002884

Shih, S.-I., Meadmore, K. L., \& Liversedge, S. P. (2012a). Aging, Eye Movements, and

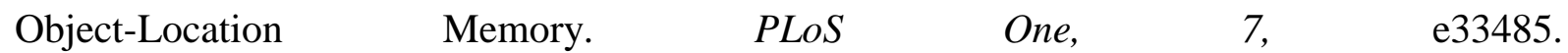
https://doi.org/10.1371/journal.pone.0033485 
Eye-tracking and organizational strategies

Shih, S.-I., Meadmore, K. L., \& Liversedge, S. P. (2012b). Using eye movement measures to investigate effects of age on memory for objects in a scene. Memory, 20, 629-637. DOI: $10.1080 / 09658211.2012 .692796$

Shing, Y. L., Werkle-Bergner, M., Brehmer, Y., Muller, V., Li, S. C., \& Lindenberger, U. (2010). Episodic memory across the lifespan: The contributions of associative and strategic components. Neuroscience and Biobehavioral Reviews, 34, 1080-1091. DOI: 10.1016/j.neubiorev.2009.11.002

Shing, Y. L., Werkle-Bergner, M., Li, S. C., \& Lindenberger, U. (2008). Associative and strategic components of episodic memory: A life-span study. Journal of Experimental Psychology: General, 137, 495-513. DOI: 10.1037/0096-3445.137.3.495

Sternberg, R. J., \& Tulving, E. (1977). Measurement of subjective organization in free recall. Psychological Bulletin, 84, 539-556. http://dx.doi.org/10.1037/0033-2909.84.3.539

Stricker, J. L., Brown, G. G., Wixted, J., Baldo, J. V., \& Delis, D. C. (2002). New semantic and serial clustering indices for the California Verbal Learning Test-Second Edition: Background, rationale, and formulae. Journal of the International Neuropsychological Society, 8, 425-435.

Stuss, D. T., Craik, F. I. M., Sayer, L., Franchi, D., \& Alexander, M. P. (1996). Comparison of older people and patients with frontal lesions: Evidence from word list learning. Psychology and Aging, 11, 387-395. http://dx.doi.org/10.1037/0882-7974.11.3.387 
Eye-tracking and organizational strategies

Taconnat, L., \& Lemaire, P. (2014). Executive functions, cognitive aging and strategic variations. Psychologie Française, 59, 89-100. https://doi.org/10.1016/j.psfr.2013.03.007

Taconnat, L., Raz, N., Toczé, C., Bouazzaoui, B., Sauzéon, H., Fay, S., \& Isingrini, M. (2009). Aging and organization strategies in free recall: The role of cognitive flexibility. European Journal of Cognitive Psychology, 21, 347-365. http://dx.doi.org/10.1080/09541440802296413

Tulving, E. (1962). Subjective organization in free-recall of unrelated words. Psychological Review, 69, 344-354. http://dx.doi.org/10.1037/h0043150

Tversky, B. (1974). Eye fixations in prediction of recognition and recall. Memory \& Cognition, 2, 275-278. https://doi.org/10.3758/BF03208995

Van der Linden, I., Rajashekar, U., Bovik, A. C., \& Cormack, L. K. (2009). Visual memory for fixated regions of natural images dissociates attraction and recognition. Perception, 38, 1152-1171. https://doi.org/10.1068/p6142

West, R. L., \& Thorn, R. M. (2001). Goal-setting, self-efficacy, and memory performance in older and younger adults. Experimental Aging Research, 27, 41-65. DOI: $10.1080 / 03610730126109$

Williams, C. C., Zacks, R. T., \& Henderson, J. M. (2009). Age differences in what is viewed and remembered in complex conjunction search. The Quarterly Journal of Experimental Psychology, 62, 946-966. http://dx.doi.org/10.1080/17470210802321976 
Eye-tracking and organizational strategies

Wingfield, A., \& Kahana, M. J. (2002). The dynamics of memory retrieval in older adulthood. Canadian Journal of Experimental Psychology, 56, 187-199. http://dx.doi.org/10.1037/h0087396

Witte, K. L., Freund, J. S., \& Brownwhistler, S. (1993). Adult age-differences in free-recall and category clustering. Experimental Aging Research, 19, 15-28. http://dx.doi.org/10.1080/03610739308253920

Witte, K. L., Freund, J. S., \& Sebby, R. A. (1990). Age-differences in free-recall and subjective organization. Psychology and Aging, 5, 307-309. http://dx.doi.org/10.1037/08827974.5.2.307

Wu, C.-C., Wick, F. A., \& Pomplun, M. (2014). Guidance of visual attention by semantic information in real-world scenes. Frontiers in Psychology, 5, article 54. https://doi.org/10.3389/fpsyg.2014.00054

Yee, E., Overton, E., \& Thompson-Schill, S. L. (2009). Looking for meaning: Eye movements are sensitive to overlapping semantic features, not association. Psychonomic Bulletin \& Review, 16, 869-874. https://doi.org/10.3758/PBR.16.5.869

Yu, Q., McCall, D. M., Homayouni, R., Tang, L., Chen, Z., Schoff, D., Nishimura, M., Raz, S., \& Ofen, N. (2018). Age-associated increase in mnemonic strategy use is linked to prefrontal cortex development. Neuroimage, 181, 162-169. DOI: 10.1016/j.neuroimage.2018.07.008 
Eye-tracking and organizational strategies

Zigmond, A. S., \& Snaith, R. P. (1983). The hospital anxiety and depression scale. Acta Psychiatrica Scandinavica, 67, 361-370. http://dx.doi.org/10.1111/j.16000447.1983.tb09716.x

Zivian, M. T., \& Darjes, R. W. (1983). Free-recall by in-school and out-of-school adults -

Performance and metamemory. Developmental Psychology, 19, 513-520. http://dx.doi.org/10.1037/0012-1649.19.4.513 
Eye-tracking and organizational strategies

\section{Annex 1}

Lists of the words and categories used to assess the participants' recall performance (with their English translations).

\begin{tabular}{|c|c|c|}
\hline \multirow[t]{2}{*}{ Non-organizable words } & \multicolumn{2}{|c|}{ Organizable words } \\
\hline & Categories & Words \\
\hline abeille (bee) & Animals & lapin (rabbit) \\
\hline orange (orange) & & canari (canary) \\
\hline guitare (guitar) & & tortue (turtle) \\
\hline luge (luge) & & renard (fox) \\
\hline cahier (notebook) & Vegetables & carotte (carrot) \\
\hline farine (flour) & & poireau (leek) \\
\hline peigne (comb) & & tomate (tomato) \\
\hline tasse (cup) & & oignon (onion) \\
\hline coton (cotton) & Body parts & jambe (leg) \\
\hline fenêtre (window) & & oreille (ear) \\
\hline tulipe (tulip) & & cheveu (hair) \\
\hline crayon (pencil) & & épaule (shoulder) \\
\hline lune (moon) & Clothing & bonnet (bonnet) \\
\hline bouche (mouth) & & manteau (coat) \\
\hline moulin (mill) & & pantalon (trousers) \\
\hline bougie (candle) & & chemise (shirt) \\
\hline piscine (swimming-pool) & Vehicles & bateau (boat) \\
\hline écran (screen) & & voiture (car) \\
\hline marteau (hammer) & & avion (airplane) \\
\hline statue (statue) & & train (train) \\
\hline
\end{tabular}


Eye-tracking and organizational strategies

Acknowledgment :

Grant from the Agence Nationale pour la Recherche (France) ANR-17-CE28-0003-02. 